\title{
A targeted population carrier screening program for severe and frequent genetic diseases in Israel
}

\author{
Joël Zlotogora ${ }^{*, 1}$, Rivka Carmi ${ }^{2}$, Boaz Lev ${ }^{3}$ and Stavit A Shalev ${ }^{4}$
}

\begin{abstract}
${ }^{1}$ Department of Community Genetics, Public Health Services, Ministry of Health and the Hebrew University Jerusalem, Israel; ${ }^{2}$ Genetics Center, Ben-Gurion University of the Negev, Beer-Sheva, Israel; ${ }^{3}$ Ministry of Health, Jerusalem, Israel; ${ }^{4}$ Genetic Institute, Ha'Emek Medical Center Afula and Bruce Rappaport School of Medicine, Technion Haifa, Israel
\end{abstract}

A national carrier screening program targeted at communities in which severe genetic diseases are present with a frequency higher than 1/1000 live births, has been in existence in Israel since 2002. Within the communities at risk, carrier screening is voluntary whereas genetic counseling and testing is provided free of charge. During the first 5 years of the program more than 13000 tests were performed, and at the end of 2007 it was offered in 35 different localities/communities for a total of $\mathbf{3 6}$ diseases. Many of the couples identified to be at risk opted for prenatal diagnosis and in two cases an affected pregnancy was terminated. In some cases the couples declined prenatal diagnosis and two of those families gave birth to an affected child. Based on the experience learnt from this targeted screening program it appears that a knowledge-based, voluntary screening program operated within the community is an effective way to provide genetic services and test referrals. The community program directed toward couples in their reproductive period does not seem to have led to stigmatization at either the individual or the community level.

European Journal of Human Genetics (2009) 17, 591-597; doi:10.1038/ejhg.2008.241; published online 24 December 2008

Keywords: population screening; consanguinity

\section{Introduction}

The Israeli Ministry of Health sponsors a 'national program for the detection and the prevention of birth defects' established in 1980. The program is provided free of charge to all citizens and is multifaceted. It is comprised of a general newborn screening, prenatal diagnosis for women at increased risk for Down syndrome and genetic diseases and a carrier screening for populations at risk for Tay Sachs disease and beta thalassemia. ${ }^{1}$ Although the newborn screening aims to prevent mental retardation, the

\footnotetext{
*Correspondence: Professor J Zlotogora, Department of community Genetics, Public Health Services, Ministry of Health, Building 67, Sheba Medical Center, Tel Hashomer, Ramat Gan 52621, Israel.

Tel: + 9722 5348432; Fax: +972 3 5355166; E-mail joelz@cc.huji.ac.il Note: This article represents the opinions of the authors and not necessarily those of the Ministry

Received 6 June 2008; revised 4 November 2008; accepted 13 November 2008; published online 24 December 2008
}

other components of the program are aimed at giving reproductive choices to the population at risk. With the characterization of the molecular basis of many other relatively frequent genetic diseases in the population, a proposal was developed to expand the genetic screening program for reproductive purposes according to the criteria first developed by Wilson and Jungner for the World Health Organization. ${ }^{2}$ Due to financial constraints, the program has not yet been incorporated into the national screening program. Currently more expansive genetic testing is being offered for a fee and the costs are partially covered by supplementary insurance plans of the Israeli health funds.

In 2002, the Ministry of Health ${ }^{3}$ expanded the free-ofcharge health basket to include a targeted carrier screening program directed at the communities in which severe genetic diseases are present with a frequency higher than $1 / 1000$ live births. This was a major first step towards the expansion of the national genetic screening program. 
A general review of this program is presented here, based on the experience of the first 5 years. Some specific parts of this program have been previously published. ${ }^{4-7}$

\section{The Israeli population and primary health care infrastructure}

In 2006 the population of Israel numbered 7116700 citizens, of whom $75.8 \%$ were Jews and 16.5\% Muslim Arabs. ${ }^{8}$ The Bedouin-Arab, residing mostly in the Negev desert, represent one-fifth of the Muslims in the country. The other groups, including mainly Christian Arabs and Druzes, each represent less than $2 \%$ of the population. For the most part, the Arabs and Druzes live in villages/tribes that were founded less than 10 generations ago by only a few individuals. The population of each of those villages/ tribes often numbers less than 10000 inhabitants. The fertility rates are very high, and in the past half-century there has been a documented 6- to 7-fold natural increase in the population of most of those communities. ${ }^{8}$ Currently in Israel there are more than 100 non-Jewish localities, with 88 having more than 2000 inhabitants. In addition, several of the large cities such as Jerusalem, Haifa and Tel Aviv-Yafo have a significant Arab population.

Middle-Eastern societies, specifically the Arab rural populations, are characterized by close family relationships. The preference of consanguinity is a result of a deeply rooted cultural custom, and even though the major religions officially discourage consanguineous marriages, they are highly prevalent in the region. In Israel more than $20 \%$ of Arab and Druze marriages are between first cousins whereas an additional $25 \%$ are between related individuals. $^{9}$ Among the Arab-Bedouin close to $70 \%$ of all marriages are consanguinous. Interreligious marriages are rare and in most localities the population is homogenous, either Muslim or Druze. When the inhabitants of a certain locality are of different religious faiths, most often they live in separate quarters.

Since the legislation of the 1995 bill of health, the entire Israeli population has been covered by national health insurance. ${ }^{1}$ Four health funds are primarily responsible for the provision and delivery of health care to the population, either through a basket of services included in the national health insurance or through supplementary insurance plans. Primary care is provided in local family clinics. In addition, there is a large network of 'maternal and child health clinics' located all over the country. This service is primarily provided by the Ministry of Health and in few locations by the health funds or by the local municipalities. In these clinics a multidisciplinary team provides primary care to healthy pregnant women and infants.

\section{Genetic diseases in Israel}

Since the creation of the State in 1948, many efforts have been made to delineate the genetic diseases in the Israeli population. ${ }^{1}$ Data about the genetic disorders present in the different communities, their clinical, metabolic and molecular basis and their frequencies were accumulated. ${ }^{2,10}$ Although among Jews the differences in the frequencies of the genetic diseases are between the communities of origin, among the non-Jews differences exist not only between the religious communities but also between the different villages/tribes. ${ }^{10}$ In the last decade, data on genetic diseases existing in Israel have been summarized and is constantly updated into current catalogs available online. ${ }^{11}$

\section{Genetic screening}

Genetic screening for Tay Sachs and thalassemia is a public health program provided free-of-charge to the Israeli populations at risk. Tay Sachs carrier screening is offered to Jews of Ashkenazi and North African descent. Thalassemia carrier screening is offered to all the Arab and Druze populations as well as to Jews originating in Iran, Iraq, Syria, Kurdistan, the Mediterranean countries and Asiatic countries of the former USSR. Although the official recommendation is to have genetic testing performed before pregnancy, many women are first made aware of the availability of genetic screening at the beginning of their pregnancy and are then referred as necessary. As a result of the screening program, Tay Sachs disease has almost disappeared among Jews in Israel, this is presumed to be due to the use of prenatal diagnosis by the general population and because of the discouragement of carrier couples' marriages within the ultra orthodox Jewish community. ${ }^{12}$ As for thalassemia, a dramatic reduction in the prevalence of the disease has been observed. ${ }^{13}$ In the late 1980s the mean number of children born each year in Israel with thalassemia was 13 among Arabs (mean 4 per 10000 live births among Jews and 50 per 10000). In the last decade the number has dropped to an average of 5 children each year (12 per 10000 among Arabs). ${ }^{14}$ Most importantly, most affected children born in the last decades were delivered to parents who, although were aware of their risk, chose not to use prenatal diagnosis and/ or pregnancy termination. ${ }^{14}$

Based on the knowledge of the distribution of genetic diseases in the population, a targeted screening program for severe genetic diseases with a frequency higher than $1 / 1000$ live births (corresponding to $6 \%$ carrier frequency for autosomal recessive diseases) was added in 2002 to the existing genetic screening program. ${ }^{3}$ As there are no known genetic diseases with such a high frequency in any of the Jewish communities, the program is in fact aimed at the non-Jewish population in Israel. The inclusion of diseases with a frequency of $1 / 1000$ live births was a first step towards the implementation of the planned comprehensive program including severe diseases up to a 
frequency of $1 / 15000$, as recommended by the Israeli Society of Medical Genetics. ${ }^{2}$ The $1 / 1000$ live births frequency was chosen so as to include most of the genetic diseases prevalent in small communities, where the program can be performed at a relatively low cost. In those delineated communities, less frequent diseases are usually limited to the close relatives of the affected individuals, therefore carrier testing, if available and indicated, is part of the genetic counseling given to the family and included in the basic services offered though the general medical insurance.

\section{Implementation of the targeted program}

The first step was the identification of the specific communities and the genetic diseases fulfilling the criteria for inclusion, namely, severe genetic diseases with early mortality or chronic severe disability and with a frequency equal to or more than $1 / 1000$ live births. ${ }^{3}$ Because of the limited experience with premarital genetic screening in the Israeli population, the screening was aimed mostly at married couples in their reproductive years. The aim of the carrier screening program was to offer reproductive choices to couples including prevention of the birth of affected children. In the case of cystic fibrosis or cerebrotendinous xanthomatosis, where early treatment significantly improves the course of the disease, an important option offered to the couples at risk was newborn diagnosis and early treatment of the disease. In two other specific cases, autosomal recessive catecholamine-induced polymorphic ventricular tachycardia in a Bedouin community and hyperoxaluria in a village near Jerusalem, the primary goal of the screening program was to identify couples at risk and allow for the early diagnosis and treatment of their affected children.

Meetings with local medical personnel and influential public figures took place to explain the aims and terms of the screening program before the actual implementation of the program in each of the localities/ communities.

Early in the course of the implementation of the targeted program, it became apparent that in several localities the set guidelines were not appropriately followed. The most significant problems encountered were the absence of regular/individual counseling sessions, screening of individuals outside the reproductive age period and, in several cases, a carrier frequency much lower than specified in the criteria. This led to the program's reorganization to assure consistent adherence to the guidelines.

In the first 3 years, the program was performed by eight separate genetic clinics and laboratories. Thereafter, the genetic counseling is being administrated regionally and is operated in five regions whereas the tests are performed in eight molecular genetics laboratories. A short explanatory movie and a pamphlet in Arabic were developed. The educational tools address the general concepts of genetic screening, using thalassemia as an example, and provide a short explanation of the respective disease(s) screened for within that particular community.

Genetic counseling and tests are provided in local clinics, by appointment and free of charge. When the screening involves a pregnant woman, the implications of a possible late diagnosis are explained to allow for an informed decision on the part of the couple as to whether to proceed with the screening. This is a two-step screening: one spouse is first examined and if found to be a carrier, he/she receives genetic counseling; and then the other spouse is examined. In the case of current pregnancy, if possible, the couple is examined simultaneously. If both spouses are found to be carriers, they receive genetic counseling that details all options available to them. A written report of the test results is sent to all individuals screened.

In each of the five regions a different geneticist is in charge of the program. This leads to minor local differences in the way the program is being implemented.

\section{Results of the screening program}

Since 2002 more than 13000 tests were performed in concordance with the guidelines of the genetic screening program. At the end of the 5-year period, the program was fully implemented in 35 different localities/communities. Almost half of the communities screened were in the ArabBedouin population in the Negev, which represent less than one-fifth of the Israeli Muslim population. The screening was also provided in 6 different Druze localities and in fact included almost half of this community in Israel. The most probable explanation of overrepresentation of Bedouins and Druze in the screened population is that these are the two commununities with the highest rates of consanguineous marriages in Israel. ${ }^{9}$ At the end of 2007, the program included a total of 36 diseases, in several cases the same disease was screened in different communities. For instance, screening for ataxia telangiectasia was provided in four different communities; in each a different founder mutation was responsible for the high prevalence of the disease. In nine of the communities more than one disease was screened with a maximum of four different diseases screened in one village (Table 1).

In almost all cases the first individual examined was a married woman, often during her pregnancy. Many couples that were both found to be carriers opted for prenatal diagnosis. In a heterozygous couple for a mutation causing spinal muscular atrophy-related disease and in another for an epidermolysis bullosa mutation, the foetuses were found affected and the pregnancies were terminated. Some carrier couples at $25 \%$ risk declined prenatal diagnosis, in particular when the screening results were obtained late in pregnancy. In one case of screened 
Table 1 A list of the diseases for which the carrier screening program was fully implemented (more than 30 individuals screened) at the end of 2007

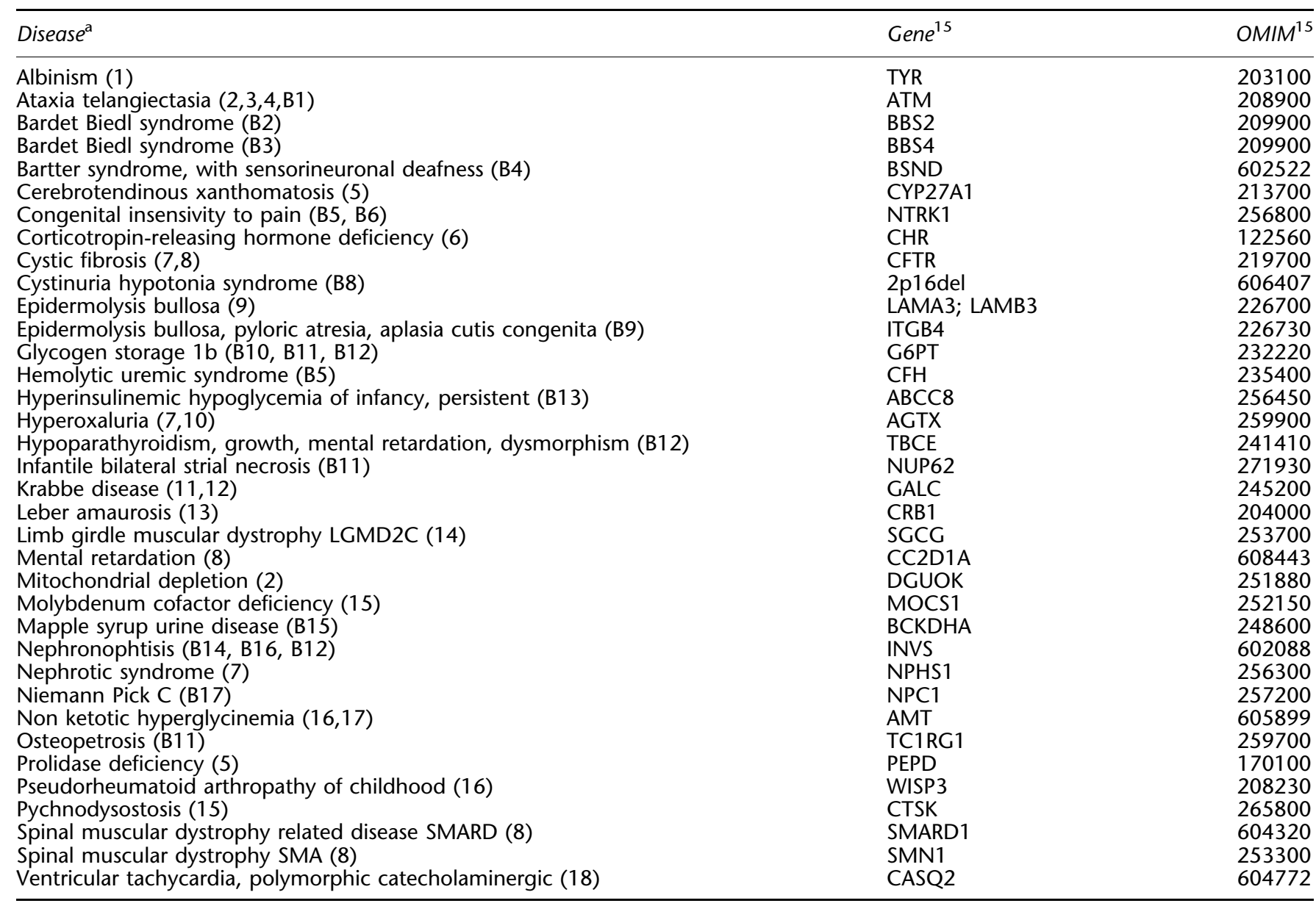

${ }^{\mathrm{a}} \mathrm{A}$ different number was given for each of the communities where the screening was provided. For the Bedouin in the Negev a B appears before the number.

heterozygous parents, a child affected with congenital nephrotic syndrome was born. In another case of an epidermolysis bullosa-affected newborn, an early diagnosis was made by CVS but the couple decided nevertheless to continue the pregnancy. Although, in general, most spouses were examined on learning that their wives were carriers, in some of the localities a high percentage of men (up to $70 \%$ in one village ${ }^{5}$ ) did not come for further counseling and examination.

Case study: an example of one of the villages included in the program

In a village of approximately 5500 people, near Jerusalem (village 7 in Table 1), most of the inhabitants are descendants of a single Arab-Muslim family who settled in this site approximately 250 years ago. Meetings with local medical personnel and influential public figures took place in the village to explain the aims and terms of the screening program. Subsequently several lectures were given to the population at large and to the local high school students. The screening services are performed in the village 'Mother and Child Well Being Clinic' where all referred/interested individuals are seen by the same medical geneticist (JZ) and by appointment. The genetic testing is also available to individuals from the village that have been referred subsequent to hospitals based genetic clinics consultations. The initiation of the screening program in this village was based on a research study that identified a cystic fibrosis carrier frequency of $8.5 \%$ from a founder mutation. ${ }^{16}$ Later, with the characterization of the molecular basis of congenital nephrotic syndrome and hyperoxaluria in this village, these tests were added to the screening. ${ }^{17}$ The unique aim of the hyperoxaluria screening was to discover couples at risk to provide early diagnosis and treatment of affected children and thereby preventing complications. In this traditional religious community, termination of pregnancy with an affected foetus, particularly in the late stages of the gestation, is 
generally not an acceptable option. In many cases the screening tests are being performed for early diagnosis and better treatment of an affected child. To date, 184 individuals were tested in this village. In 2003, 26\% of the women giving birth had genetic counseling before or during the pregnancy (26 out of 100 women). This number increased in 2007 to $45.8 \%$ (54 out of 118 women). In most cases where the counseling and testing was performed during pregnancy, the women did not have any history of genetic problems in their first- or second-degree relatives. In addition, in 18 cases the genetic counseling was preconceptional and in 5 cases premarital. Among the 184 individuals screened, the carrier frequencies were $9.2 \%$ for cystic fibrosis, 9.3\% for nephrotic syndrome and $8.1 \%$ for hyperoxaluria. In the 30 couples where the women were diagnosed as carriers of either cystic fibrosis or nephrotic syndrome, 10 husbands declined further screening. Two out of three couples heterozygous for cystic fibrosis had severe fertility problems and were referred for IVF. Infertility seems to be frequent in the village, in part probably due to a high frequency of the 5T allele of CFTR. ${ }^{16}$ This polymorphism is not part of the mutations screened in the program but was examined in the two couples mentioned because of their infertility. The polymorphism was not found in either of the husbands. Because of the $25 \%$ risk for cystic fibrosis the two couples that underwent IVF, opted for preimplantation genetic diagnosis (PGD), and a healthy child was born in each case. The third heterozygous couple had a prenatal diagnosis and a healthy newborn.

In one case where the woman was found to be a carrier of congenital nephrotic syndrome, maternal serum $\alpha$-fetoprotein was detected at a very high level, suggesting an affected foetus. The husband was subsequently also found to be a carrier. The couple declined prenatal diagnosis and an affected child was born.

\section{Comparison between the targeted Israeli program and other genetic screening programs}

With the decreased mortality and morbidity due to infectious diseases and prematurity, congenital malformations and genetic diseases have become a major cause of morbidity and mortality among infants and children. As a result, programs aimed at the prevention of genetic diseases, particularly for thalassemia were developed for the most part, in collaboration with the World Health Organization. In countries such as Iran, Saudi Arabia and other Muslim nations where thalassemia is highly prevalent, premarital screening is mandatory. ${ }^{18-22}$ Although a premarital certificate that the tests have been performed is obligatory, the results are not taken into account when issuing a marriage licence. In Iran, most of the heterozygous couples decided to marry and the incidence of the disease was not significantly affected. This eventually lead to the enactment of a law, based on principles put forward by a religious decree (Fatwa), allowing for the termination of pregnancies before and up to the 120th day of pregnancy in cases of severe disease in the fetus. ${ }^{22}$

A different approach to premarital screening was adopted in Cyprus. Thalassemia screening is required but the results of the screening do not preclude a religious marriage within the church. ${ }^{18}$ The Jewish ultra orthodox community also requires genetic screening before marriage. In this community, marriages are prearranged and the results of the genetic screening is one of the decisive factors as to whether the match-making will proceed (Dor Yeshorim). ${ }^{12}$ In each of these two religious communities, the programs resulted in a highly significant reduction in the prevalence of the diseases screened: in Cyprus because of the use of prenatal diagnosis by couples at risk, and in the Ultra orthodox Jewish community by avoidance of marriages between carriers.

Several ethical questions may be raised concerning the mandatory screening programs, but experience seems to demonstrate that if the programs are confidential, culturally appropriate allowing individual freedom of choice as to how to proceed; the programs are well accepted by the screened population.

In addition to those carrier screening programs, pilot studies examined the feasibility of screening programs for relatively frequent diseases in the general population such as cystic fibrosis and fragile $X$ syndrome. ${ }^{23-27}$ In the United States, preconceptional cystic fibrosis carrier screening is recommended and performed using a consensus panel of mutations. ${ }^{26}$

For obvious reasons, it is expected that mandatory premarital genetic screening will be difficult or even impossible to implement in western societies in general and in particular if minorities are to be targeted. In addition, there is the contemporary secular problem of pregnancies that often precede formal marriages. A better alternative in such communities is a voluntary preconceptional screening together with an appropriate educational program targeting young adults. Examples of high schoolbased educational programs that included genetic testing have been reported to be very successful in Canada and in Australia. ${ }^{28,29}$ Although high schools offer a convenient setting for genetic screening and it has many advantages, many questions have been raised about school-based blood testing in adolescents. ${ }^{30,31}$ As all the evaluations of these high school screening programs found that education played a critical role in providing for informed testing decisions, a better solution would be to leave the student with the option of when to perform the test outside of the convenient school-binding context. ${ }^{30}$

In Israel, voluntary carrier genetic screening for Tay Sachs disease and thalassemia has been successfully provided for more than 25 years. This program has served as a model for the newly established targeted genetic 
screening aimed at severe genetic diseases with a frequency higher than $1 / 1000$ live births. In this new program pregnant women from the designated communities at risk are given the option of having genetic testing and when appropriate are referred for the actual performance of the test. A major difference between the screening program for Tay Sachs or thalassemia and the community-targeted program is that the later including genetic counseling is offered at the place of residence. The screening is offered to every couple in the designated at-risk community, regardless of whether any genetic disease actually exists in the family. This was purposely done to reduce possible stigmatization. Indeed, the program has been well accepted on both the individual and the community levels by different communities regardless of religions, traditions or socio-economical status. Over time we have observed improving knowledge of the genetic tests available, both among medical personnel and laymen in the communities. Although there has not been any systematic study, we are not aware of any program-related stigmatization. It has been our experience that the establishment of genetic clinics within the community increased awareness and accessibility and thus the demand for premarital counseling and preconceptional screening. ${ }^{32}$

As already noted, in several of the screened communities, husbands of screened women carriers did not come for counseling and testing. This may probably indicate a lack of thorough understanding of the aims of the program because initial participation infers the potential need to examine the spouse. Obviously, additional educational efforts are needed.

Many of the couples at risk that were discovered by the program opted for the prevention of the birth of an affected child. However, prenatal diagnosis and termination of pregnancy of an affected foetus is often very problematic. Two heterozygous couples with fertility problems used PGD within the framework of IVF and thus pregnancies with affected children were prevented. With the recent advancements in the use of PGD and the intention to include it in the Israeli basket of health services, this mode of genetic diagnosis is becoming a significant alternative for many families at risk for whom termination of pregnancy is not a religiously, traditionally or culturally acceptable option.

From the experience of the targeted screening program presented here it seems that voluntary screening within the community is an effective alternative to mandatory screening. However, for an individual to have a truly free choice of reproductive options she or he must be properly informed and knowledgeable of the program before the first pregnancy. Therefore, for such a voluntary program to be effective it must be accompanied by an extensive educational program that probably needs to start in high school and a concurrent community outreach program that includes as wide a circle in the community as possible $^{30}$. As a first step toward this goal we began in 2007 to implement a pilot education program in high schools.

\section{Acknowledgements}

We thank all the geneticists involved in the screening program.

\section{References}

1 Chemke J, Zlotogora J: Genetic services in Israel. Eur J Hum Genet 1997; 5 (Suppl 2): 105-111.

2 Zlotogora J, Leventhal A: Screening for genetic disorders among Jews. How should the Tay Sachs screening program be continued? IMAJ 2000; 2: 665-667.

3 Shalev AS, Carmi R, Leventhal A, Zlotogora J: A program for prevention of genetic diseases in Israel. Harefuah (in Hebrew) 2003; 142: $792-794$.

4 Basel-Vanagaite L, Taub E, Halpern GJ et al: Genetic screening for autosomal recessive nonsyndromic mental retardation in an isolated population in Israel. Eur J Hum Genet 2007; 15: 250-253. Epub 2006 Dec 6.

5 Basel-Vanagaite L, Taub EJ, Drasinover V et al: Genetic carrier screening for spinal muscular atrophy and spinal muscular atrophy with respiratory distress in an isolated population in Israel. Genet Test 2008; 12: 53-56.

6 Khayat M, Shapira H, Kfir N, Gurevitz I, Mandel H, Falik-Zaccai TC: Four Metabolic Disorders in a Genetic Isolate: Population Screening and Disease prevention. [Abstract 1702]. Presented at the annual meeting of The American Society of Human Genetics, October 2003, Los Angeles, California. Available at: http://www.ashg. org/genetics/abstracts/abs03/f1702.htm.

7 Mishori-Dery A, Birk OS, Elbedour K, Yunis A, Beit-Or H, Shoham-Vardi I: Screening for Carriers of Genetic Diseases in High Risk Populations - Designing a Culturally Appropriate Implementation Program. Abstract, 3rd International Conference on Birth Diseases and Disabilities Rio de Janeiro, Brazil, 17-21 June 2007.

8 Statistical Abstracts of Israel. Jerusalem 2007. Available online www1.cbs.gov.il

9 Vardi-Saliternik R, Friedlander Y, Cohen T: Consanguinity in a population sample of Israeli Muslim Arabs, Christian Arabs and Druze. Ann Hum Biol 2002; 29: 422-431.

10 Zlotogora J: The molecular basis of autosomal recessive diseases among the Palestinian Arabs. Am J Med Genet 2002; 109: $176-182$.

11 The Israeli Mutation Database www.goldenhelix.org/israeli (accessd on the 4 of December 2008).

12 Ekstein J, Katzenstein H: The Dor Yeshorim story: communitybased carrier screening for Tay-Sachs disease. Adv Genet 2001; 44: 297-310.

13 Koren A, Zalman L, Palmor $\mathrm{H}$ et al: The prevention programs for beta thalassemia in the Jezreel and Eiron valleys: results of fifteen years experience. Harefuah (in Hebrew) 2002; 141: 938-943.

14 Department of Community Genetics, www.health.gov.il/genetics (accessd on the 4 of December 2008).

15 http://www.ncbi.nlm.nih.gov/sites/entrez?db = OMIM (accessd on the 4 of December 2008).

16 Chiba-Falek O, Nissim-Rafinia M, Argaman Z et al: Screening of CFTR mutations in an isolated population: identification of carriers and patients. Eur J Hum Genet 1998; 6: 181-184.

17 Frishberg Y, Ben-Neriah Z, Suvanto M et al: Misleading findings of homozygosity mapping resulting from three novel mutations in NPHS1 encoding nephrin in a highly inbred community. Genet Med 2007; 9: 180-184.

18 Kalokairinou EM: The experience of beta-thalassaemia and its prevention in Cyprus. Med Law 2007; 26: 291-307.

19 Christianson A, Streetly A, Darr A: Lessons from thalassaemia screening in Iran. BMJ 2004; 329: 1115-1117. 
20 Alhamdan NA, Almazrou YY, Alswaidi FM, Choudhry AJ: Premarital screening for thalassemia and sickle cell disease in Saudi Arabia. Genet Med 2007; 9: 372-377.

21 Samavat A, Modell B: Iranian national thalassaemia screening program. BMJ 2004; 329: 1134-1137.

22 Hesketh T: Getting married in China: pass the medical first. BMJ 2003; 326: 277-279.

23 Loader S, Caldwell P, Kozyra A et al: Cystic fibrosis carrier population screening in the primary care setting. Am J Hum Genet 1996; 59: 234-247.

24 Witt DR, Schaefer C, Hallam P et al: Cystic fibrosis heterozygote screening in 5,161 pregnant women. Am J Hum Genet 1996; 58: $823-835$

25 Fanos JH, Spangner KA, Musci TJ: Attitudes toward prenatal screening and testing for Fragile X. Genet Med 2006; 8: $129-133$.

26 American College of Obstetricians and Gynecologists, American College of Medical Genetics: Preconception and Prenatal Carrier Screening for Cystic Fibrosis: Clinical and Laboratory Guidelines.
Washington, DC: American College of Obstetricians and Gynecologists, 2001.

27 Metcalfe S, Jacques A, Archibald A et al: A model for offering carrier screening for fragile $\mathrm{X}$ syndrome to nonpregnant women: results from a pilot study. Genet Med 2008; 10: 525-535.

28 Mitchell JJ, Capua A, Clow C, Scriver CR: Twenty-year outcome analysis of genetic screening programs for Tay-Sachs and betathalassemia disease carriers in high schools. Am J Hum Genet 1996; 59: 793-798.

29 Gason AA, Metcalfe SA, Delatycki MB et al: Tay Sachs disease carrier screening in schools: educational alternatives and cheekbrush sampling. Genet Med 2005; 7: 626-632.

30 Frumkin A, Zlotogora J: Genetic screening for reproductive purposes at school: is it a good strategy? Am J Med Genet A 2008; 146: 264-269.

31 Ross LF: Heterozygote carrier testing in high schools abroad: what are the lessons for the U.S.? J Law Med Ethics 2006; 34: 753-764

32 Zlotogora J, Barges S, Bisharat B, Shalev SA: Genetic disorders among Palestinian Arabs. 4. Genetic clinics in the community. Am J Med Genet 2006; 140A: 1644-1646. 